\title{
Review
}

Journal of Innate

Immunity
J Innate Immun 2017;9:262-270

DOI: $10.1159 / 000455100$
Received: October 25, 2016

Accepted after revision: December 14, 2016

Published online: January 27, 2017

\section{Participation of Necroptosis in the Host Response to Acute Bacterial Pneumonia}

\author{
Danielle Ahn ${ }^{a} \quad$ Alice Prince $^{a, b}$ \\ Departments of a Pediatrics and ${ }^{\mathrm{b}}$ Pharmacology, Columbia University Medical Center, New York, NY, USA
}

\section{Keywords}

Necroptosis · Bacterial pneumonia · Cellular death

\begin{abstract}
Common pulmonary pathogens, such as Streptococcus pneumoniae and Staphylococcus aureus, as well as the hostadapted pathogens responsible for health care-associated pneumonias, such as the carbapenem-resistant Klebsiella pneumoniae and Serratia marcecsens, are able to activate cell death through the RIPK1/RIPK3/MLKL cascade that causes necroptosis. Necroptosis can influence the pathogenesis of pneumonia through several mechanisms. Activation of this pathway can result in the loss of specific types of immune cells, especially macrophages, and, in so doing, contribute to host pathology through the loss of their critical immunoregulatory functions. However, in other settings of infection, necroptosis promotes pathogen removal and the eradication of infected cells to control excessive proinflammatory signaling. Bacterial production of pore-forming toxins provides a common mechanism to activate necroptosis by diverse bacterial species, with variable consequences depending upon the specific pathogen. Included in this brief review are data demonstrating the ability of the carbapenem-resistant ST258 K. pneumoniae to activate necroptosis in the setting of pneumonia, which is counterbalanced by their sup-
\end{abstract}

pression of CYLD expression. Exactly how necroptosis and other mechanisms of cell death are coregulated in the response to specific pulmonary pathogens remains a topic of active investigation, and it may provide potential therapeutic targets in the future.

(c) 2017 S. Karger AG, Basel

\section{Introduction}

Understanding the molecular pathways responsible for cell death has provided important insights into the pathogenesis of many types of human disease, including bacterial infection [1]. Tissue destruction, caused by bacterial products, proteases, DNAses, collagenases, and toxins, which results in frank necrosis, was long recognized as a major mechanism of pathology. Over the past several decades, specific pathways of "programmed cell death" have been clearly delineated. Historically, apoptosis was first identified as a regulated series of biochemical events resulting in typical cellular morphology, with blebbing of the cytoplasmic membrane and chromatin condensation $[2,3]$. This leads to the overall involution and death of cells that occur without an inflammatory response. Annexin V, a cellular marker of apoptotic cells, labels the phosphotidylserine residues characteristically 
showing from exposed cellular membranes [4]. While a critical component of normal growth and development, apoptosis was also identified as a mechanism to eliminate infected cells, particularly by viruses [5]. The activation of apoptosis through TNF signaling and the caspases responsible were recognized, further defining its role in infection [6].

It became clear that there were additional mechanisms of programmed cell death that result in very distinct pathological outcomes. Instead of membrane blebbing and cellular involution, cells underwent what was then called "programmed necrosis" but is now termed necroptosis. This process has loss of cytoplasmic membrane integrity, is caspase-independent, and, in some settings, is highly inflammatory with the release of immunogenic intracellular components [7]. Initial studies described the important role of this cascade in the clearance of viral infection. Recognition of cytosolic viral DNA by activated TLR3/ TRIF, DAI, and the interferons (IFNs) induced necroptosis and eliminated virally infected cells. Shared by receptor-interacting protein (RIP), TRIF and DAI proteins, the common RIP homotypic interaction motif (RHIM) domains enabled their interaction in necroptosis [8]. Identification of the components of the necroptosis pathway was achieved through the use of biochemical inhibitors, the necrostatins that target RIPK1 [9], and later on, necrosulfonamide (NSA) that blocks the effector mixedlineage kinase domain (MLKL). Subsequent studies indicated that intracellular bacterial infection also resulted in the activation of IFNs and necroptosis [10].

Identification of necroptotic cells has been challenging, as there are no accepted cellular markers such as annexin V. Instead, cytotoxicity associated with MLKL activation is now required to define cell death mediated by this specific cascade [11]. Through a series of studies using biochemical inhibitors of apoptosis and the analysis of the mice lacking specific members of the RIP kinase family, the components of necroptosis have been identified. As amply reviewed elsewhere [1, 7, 10, 12], necroptosis is mediated by the necrosome, which is activated by TLR/TRIF or TRADD signaling to induce RIPK1 phosphorylation. This targets RIPK3 that, in turn, phosphorylates MLKL, a pseudokinase that multimerizes to expose an N-terminal motif that interacts with phospholipids at the cell membrane, forms a pore, and ultimately leads to cell death $[13,14]$. Upstream activators include the TNF pathway and TLRs, especially those that recognize intracellular ligands such as DNA and LPS. Numerous interacting pathways link apoptosis and necroptosis with their relative participation dependent upon the availability of caspase-8 [15]. In the absence of caspase-8, TNF signaling as well as that initiated by specific TLRs can activate necroptosis [16]. Recent studies demonstrate that RIPK1 and RIPK3 interactions drive inflammatory cytokine production through TLR4-TRIF signaling that is independent of necroptosis [17]. Thus, this complex is critical in determining the outcome of the proinflammatory signaling that is initiated by TLR or the TNF cascade.

In addition to cell death, the RIP kinases have a major role in immunoregulation, both pro- and anti-inflammatory [18]. Analysis of knockout mice lacking components of the necrosome demonstrated excessive inflammatory responses, or the mutations were embryonically lethal, due to hepatic and skin inflammation, indicating a key regulatory role for the proteins involved in necroptosis. It is important to recognize that RIPK1 also has scaffold functions that are independent of its kinase activity [19]. The posttranslational regulation of the RIP kinases is complex and involves numerous interacting phosphatases, ubiquitinylating enzymes, and caspases, as reviewed recently [20]. The ubiquitylation of RIPK1 promotes the activation of NF- $\kappa B$, which is mediated through NEMO and TAK1 [21]. RIPK1 is also a target for the deubiquitylating enzyme CYLD and can negatively regulate the activation of NF- $\kappa B$. Both RIPK1 and RIPK3 are targets of caspase- 8 , which cleaves the deubiquitinase CYLD and prevents the initiation of necroptosis [22]. As recently reviewed, it is evident that RIPK3 has a central role in the regulation of inflammatory signaling, and, accordingly, is controlled through multiple mechanisms that include its phosphorylation, ubiquitination, and interactions with available caspases [23].

\section{Necroptosis and Pyroptosis}

In contrast to the embryonic lethality of $R i p k 1^{-/-}$mice, $R i p k 3^{-/-}$mice are viable and healthy, and have been used to study the role of necroptosis in bacterial infection [24, 25]. However, as noted above, RIPK3 has multiple interactions within the cell, and its deletion may affect more than necroptosis. Inflammasome activation is linked to RIPK3, which spatially coordinates caspase-1-dependent cleavage of the proinflammatory cytokines pro-IL $1 \beta$ and pro-IL-18 [26]. The activation of the inflammasome results in caspase-1-mediated cell death, or pyroptosis, which is highly proinflammatory as it releases the intracellular components of the targeted cell and also IL- $1 \beta$ and IL-18. In contrast to necroptosis, the effector of inflammasome-mediated cell death is gasdermin $\mathrm{D}$, a substrate for caspase-1 and caspase-11 [27]. The participa- 
tion of the inflammasome and the activation of pyroptosis in cell death is often caused by bacterial toxins, also observed with necroptosis [25]. Pro-IL-1 $\beta$ can be processed by both caspase- 8 and caspase- 1 in an RIPK3-dependent fashion [16]. However, the biochemical interaction between RIPK3 and the inflammasome has not been clearly delineated.

\section{Necroptosis in Bacterial Infection}

The activation of cell death has long been recognized as an important mechanism of pathogen clearance, whether cell death is mediated by apoptosis, necroptosis, or pyroptosis. The consequences of the discrete mechanisms of cell death may be quite distinct depending upon whether the targeted cell contains a pathogen that must remain intracellular to survive, as in many viral infections, or whether cell death releases a viable pathogen in the case of intracellular bacteria. These microbes can then be efficiently killed by another professional phagocyte, such as a neutrophil, or induce cellular death through necroptosis [28]. Intracellular bacterial infections that result in the release of DNA, sensed by DAI or TLR9, or ligands that can activate TRIF and IFN signaling have been found to induce necroptosis, based upon the established interactions of the RHIM-containing proteins, RIPK1, RIPK3, TRIF, and DAI $[8,29]$. If critical regulatory cells such as macrophages [30] or T cells [31] are also eliminated, the net result is excessive inflammation. Alternatively, bacterial virulence factors such as NleB, a type III secretion system effector of Escherichia coli, can actively impair the induction of necroptosis by inhibiting death domain formation [32]. The resultant inflammatory tone set by necroptosis in the setting of bacterial infections is therefore likely organism-dependent.

\section{Necroptosis in Macrophages}

Intracellular bacterial pathogens that target macrophages can induce pathological responses through necroptosis and promote the loss of this critical component of the immune response. In a model of Salmonella typhimurium, macrophage loss through type I IFN-dependent necroptosis was observed, and this resulted in the enhanced systemic proliferation of the organisms, a response that did not occur in Ifnar ${ }^{-1-}$ mice [29]. Mycobacterium tuberculosis also targets macrophages with an effective host response to infection dependent upon TNF production [10]. Activation of RIPK1 and RIPK3 were found to be important in the control of $M$. tuberculosis infection through TNF signaling that led to mitochondrial ROS production and sphingomyelinase activity [33]. In the case of $M$. tuberculosis, cellular death by these pathways is advantageous to the organism as the bacteria are allowed to replicate intracellularly without being killed and released after host cell death.

\section{Induction of Necroptosis by Diverse Pore-Forming Bacterial Toxins}

Several studies have demonstrated that a variety of bacterial pore-forming toxins kill host cells, particularly macrophages, through necroptosis [34]. These toxins include listeriolysin, produced by the intracellular pathogen Listeria monocytogenes, and also the toxin associated with uropathogenic E. coli. More relevant to pulmonary infection, Streptococcus pneumoniae pneumolysin and the pore-forming toxin ShlA produced by Serratia marcescens in a murine model of pneumonia were potent activators of necroptosis. The investigators documented that the cytotoxicity associated with these toxins was not caspase-dependent and that mice lacking MLKL were protected from severe pneumonia. As was shown with $S$. aureus infection, macrophages were especially susceptible to necroptosis induced by these pore-forming toxins [25].

These studies carefully documented that $\mathrm{Mlkl}^{-1-}$ and $R i p k 3^{-/}$mice were protected from the cytoxocitiy associated with these toxins, and that caspase- 1 and caspase- 8 were not required to induce cell death. However, as might be predicted from the biochemical studies of RIPK3NLRP3 interactions, there were associations observed between the necroptosis and pyroptosis pathways. These authors also suggested a role for MyD88 in mediating this cytotoxicity. Of note, each of the different bacterial strains and toxins had a somewhat distinct degree of interaction with inflammasome components. These very interesting results confirm that the activation of necroptosis and the stimulation of inflammasome components by clinically important bacterial pathogens may be linked, but no suggestion was made of a unified mechanism to explain how this occurs.

\section{Necroptosis in Staphylococcus aureus Pneumonia}

In contrast to Salmonella that actively infects macrophages and M. tuberculosis that persists in intracellularly promoting granuloma formation, Staphylococcus aureus 
is usually an acute extracellular pathogen, frequently associated with severe pneumonia following influenza, but also in otherwise-well individuals. S. aureus was shown to stimulate a type I IFN response in macrophages, mediated through TLR9 and TRIF [35] suggesting the possibility that this organism could also activate RIPK1/ RIPK3 responses. Ifnar ${ }^{-/}$mice have been observed to have significantly improved outcomes from $S$. aureus pneumonia [36], so it was hypothesized that some component of this pathway is associated with pathological outcomes from infection. Analysis of the clearance of S. aureus from Ripk $^{-/-}$mice compared with wild-type (WT) controls indicated the retention of a much greater population of immunoregulatory macrophages, i.e., those expressing CD200R and CD206 in the mice lacking Ripk3. These anti-inflammatory macrophages usually serve to reduce inflammation in the airways, and their survival in Ripk $^{-/-}$lungs is likely a major reason for more efficient clearance of $S$. aureus from infected lungs [25]. Both human and murine macrophages were shown to be highly susceptible to the necroptosis initiated by S. aureus, demonstrated by the inhibition of cell death by necrostatin, an inhibitor of RIPK1, or NSA, an inhibitor of MLKL.

The mechanism(s) through which $S$. aureus induces necroptosis is entirely toxin-dependent [25]. Despite the association of RIPK1/RIPK3 and TRIF signaling activated by $S$. aureus infection, the IFN pathway has not been found to be responsible for the activation of necroptosis by $S$. aureus. Instead, necroptosis is activated by exoproducts released into the culture supernatant. The comparison of several mutants lacking expression of specific pore-forming toxins has indicated a role for the a-toxin (Hla), phenol-soluble modulins, and LukAB, but not PVL in necroptosis. Since each of these toxins has a discrete receptor, it is difficult to identify a common pathway by which necroptosis is activated. However, the ability of these secreted toxins to activate necroptosis in many different types of immune cells, including neutrophils [28] and $\mathrm{T}$ cells, may contribute to their major role in pathogenesis. It is important to recognize that although the neutrophil population may be readily replenished from bone marrow stores, the loss of immunoregulatory cells through necroptosis may have a significant effect on the regulation of the overall inflammatory response. These studies also documented that, at least for S. aureus, the induction of necroptosis did not necessarily result in staphylococcal killing, in contrast to the caspase-1-dependent mechanisms of cell death associated with decreased bacterial viability [37].

Necroptosis in Bacterial Pneumonia

\section{The Role of Necroptosis in Systemic Infection}

Whereas the bacterial induction of necroptosis via the staphylococcal pore-forming toxins is detrimental in the lung due to the elimination of substantial numbers of anti-inflammatory macrophages, the role of necroptosis in systemic infection can be quite different. Models of infection that used RIPK1 inhibition with necrostatin-1, or the deletion of Ripk3 provided protection in TNF-induced inflammation, or in a cecal ligation and puncture model of septic shock [37]. Systemic inflammation was observed in pathology studies of Ripk $1^{-/-}$mouse embryos and neonates [38], and it was demonstrated that RIPK1 controls the excessive inflammatory signaling driven by RIPK3MLKL signaling. Ripk $3^{-/-}$mice have been shown to have deficient induction of IL-1 $\beta$, IL-23, and IL-22 in the setting of injury-induced inflammation [23]. The appreciation that RIPK3 has multiple binding targets must be considered when interpreting these findings, but there is substantial data confirming an overall anti-inflammatory role for necroptosis in systemic infection.

To determine if systemic infection with $S$. aureus, which often accompanies pneumonia, similarly induces a protective necroptotic response, the clearance of MRSA from the blood, and the overall survival of WT, Ripk $3^{-/-}$, and $M l k l^{-1-}$ mice were compared along with mice treated with the RIPK1 and RIPK3 inhibitors [39]. In this model, necroptosis had a protective, anti-inflammatory effect with enhanced staphylococcal clearance. Of note, the $\mathrm{Ripk}^{-{ }^{--}}$mice had a better outcome than the $\mathrm{Mlkl^{-1- }}$ mice, likely due to the link between RIPK3 and inflammatory signaling.

\section{Necroptosis in Host-Adapted Pathogens: Carbapenem-Resistant Klebsiella pneumoniae}

The role of necroptosis in the pathogenesis of gramnegative pneumonia is not well understood. We provide recent data to demonstrate some of the challenges of establishing exactly how the induction of necroptosis by the common respiratory pathogen $K$. pneumoniae contributes to pathogenesis. The prototypic strain of $K$. pneumoniae ATCC 43816 (KPPR1) has been shown to suppress the expression of CYLD [40], a deubiquitinase that targets the effectors of necroptosis RIPK1 and RIPK3. Although CYLD suppression has been shown to decrease the induction of NF- $\kappa B$, its role in activating cell death with $K$. pneumoniae infection has not established. We performed a series of experiments to determine if the 
Fig. 1. CRKP stimulates necroptosis. Immunoblot for CYLD (Sigma), a deubiquitinase targeting RIP kinases, of the monocyte (THP-1) cell lysate of cells infected with KP35 (MOI 10) (a) compared to the media (MED) control or lung homogenate from C57BL/6J mice $(n=4)$ infected with KP35 $\left(10^{8} \mathrm{CFU}\right)(\mathbf{b})$ for $96 \mathrm{~h}$. THP-1 cells were pretreated for $1 \mathrm{~h}$ with DMSO vehicle, NSA, or ZVAD, and then infected with either K. pneumoniae ATCC 43816 (KPPR1) or KP35 (MOI 10) for $2 \mathrm{~h}$, prior to the addition of gentamicin. Supernatants were collected at $24 \mathrm{~h}$ and tested for LDH activity (c) or IL- $1 \beta$ (d) by ELISA. ${ }^{*} p<0.05$, *** $p<0.005$, ${ }^{* * * *} p<0.0001$, 1-way ANO$\mathrm{VA}$, Tukey test for multiple comparisons.

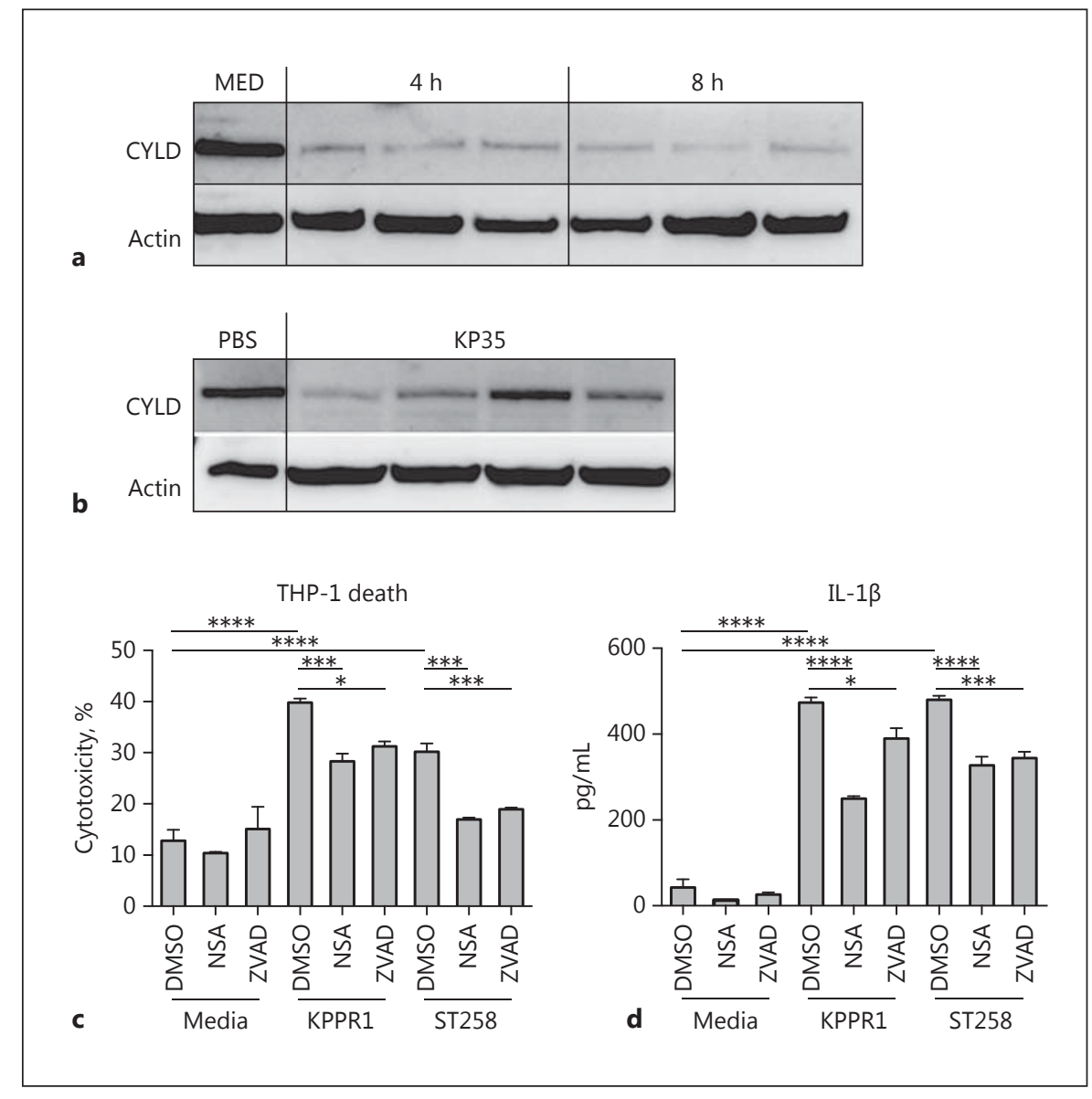

more common ST258 K. pneumoniae isolates [41, 42], currently a major cause of infection in US hospitals, similarly decrease CYLD production, and if the subsequent suppression of necroptosis contributes to the pathogenesis of respiratory infection.

We selected a representative K. pneumoniae ST258 clinical isolate 35 (KP35) comprehensively studied elsewhere [42] from a patient with bacteremia [43]. The predicted suppression of CYLD by KP35 was observed in both the monocyte/macrophage THP-1 cell line and in whole-lung homogenates of infected mice (Fig. 1a, b). This reduction in CYLD should prevent RIPK1/RIPK3 interactions and inhibit cytotoxicity through necroptosis. In vitro studies demonstrated significant protection from cytotoxicity following NSA treatment, which inhibits MLKL, showing that carbapenem-resistant Klebsiella pneumoniae (CRKP) activates necroptosis in THP-1 cells (Fig. 1c). The pan-caspase-inhibitor ZVAD also prevented cytotoxicity, and both inhibitors decreased K. pneumoniae induction of IL-1 $\beta$ production (Fig. 1d). Thus, in the case of
KP35 infection, the induction of necroptosis is not due to increased CYLD, it is rather that the previously observed inhibition of necroptosis by CYLD was not detected.

To directly address whether the induction of necroptosis contributes to the pathogenesis of pneumonia by CRKP strains, outcomes in WT and Ripk $3^{-/-}$mice infected with KP35 were compared. Ripk $3^{-/-}$mice experienced improved bacterial clearance from both the bronchoalveolar fluid (BALF) $(p=0.058)$ and the lung $(p=0.007)$ at $96 \mathrm{~h}$ of infection (Fig. 2a, b). There was a trend toward decreased TNF in the BALF of the Ripk $3^{-/-}$mice (Fig. 2c). However, these findings were not associated with significant changes in the recruitment of immune cells, neutrophils, macrophages/monocytes, or dendritic cells (Fig. 2d-f). Experiments were also performed with mice expressing RIPK1 lacking kinase activity but preserving its critical scaffold function, i.e., RIPK1 kinase-dead mice. At $96 \mathrm{~h}$ after infection, there were no significant differences in KP35 clearance between the WT and RIPK1 kinase-dead mice (Fig. 2g, h). Thus, in contrast to S. aureus 


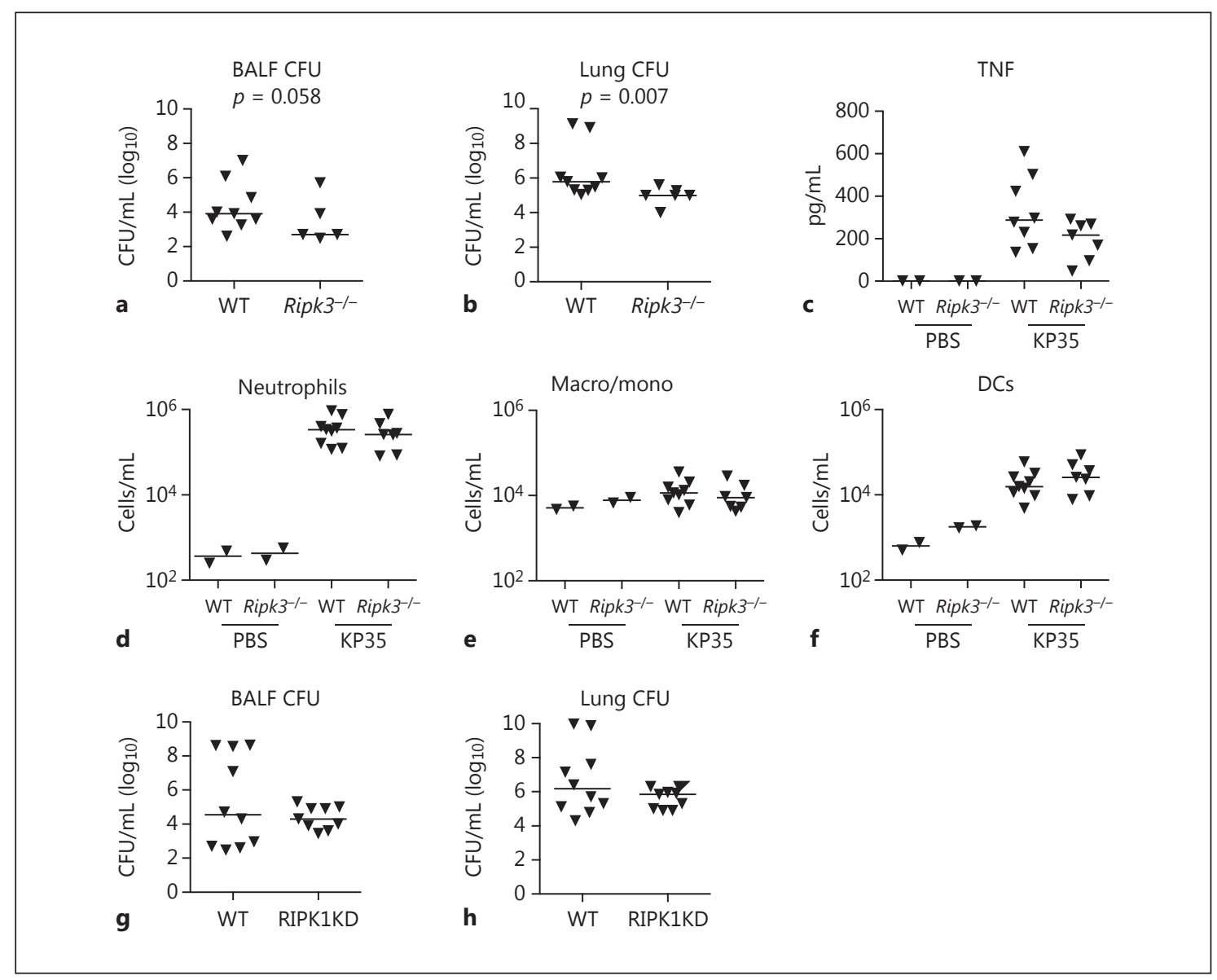

Fig. 2. Improved clearance of KP35 in Ripk $3^{-/-}$mice. WT C57BL/6J and Ripk $3^{-1-}$ mice (Genetech) were infected with KP35 (10 $\left.{ }^{8} \mathrm{CFU}\right)$ for $96 \mathrm{~h}$, and bacterial clearance was quantified in the bronchoalveolar lavage fluid (BALF) (a) or lung homogenate (b) as previously described [42]. c TNF levels in BALF were measured by ELISA (BioLegend). Analysis of cell populations in BALF was conducted using BD FACSCalibur as previously described [45]. Neutrophils (Ly6G/C $\left.{ }^{+} \mathrm{MHCII}^{\text {neg}}\right)(\mathbf{d})$, macrophage/monocytes (Mac- ro/mono) (CD11 $\left.\mathrm{c}^{+} \mathrm{MHCII}^{\text {neg }}\right)$ (e), and dendritic cells (DCs) $\left(\mathrm{CD} 11 \mathrm{c}^{+} \mathrm{MHCII}^{\mathrm{pos}}\right)(\mathbf{f})$ in BALF of KP35-infected mice versus PBS controls. KP35 $\left(10^{8} \mathrm{CFU}\right)$ clearance at $96 \mathrm{~h}$ in C57BL/6J and RIPK1KD mice (GSK Biologicals SA), BALF (g), or lung homogenate $(\mathbf{h}) . \mathrm{KD}$, kinase-dead. $p$ values as already indicated (MannWhitney $U$ test). Animal experiments were performed in accordance with the guidelines of the IACUC at Columbia University (protocol No. AAAG9307). infection, in which toxin-associated necroptosis plays an important role in pathogenesis, with $K$. pneumoniae, which does not produce pore-forming toxins [44], necroptosis seems less critical in the overall outcome after pneumonia, but may contribute to the substantial immune dysregulation seen in these infections.

\section{Conclusions}

As we have reported, many different airway pathogens can stimulate necroptosis to different degrees and in different cells. For the common airway pathogens $S$. aureus and S. pneumoniae, the production of toxins that activate necroptosis is an important component of "virulence." Mutants lacking these toxins cause less airway damage, kill fewer immune cells, and are more efficiently cleared. The immune cells targeted by pore-forming toxins, such as staphylococcal Hla or pneumococcal pneumolysin undergo necroptosis as well as pyroptosis, releasing proinflammatory cytokines but also eliminating infected cells from the airway.

The consequences of necroptosis induced by 2 different organisms that generate pore-forming toxins can be quite different (Fig. 3). Both S. marcescens and S. aureus toxins have similar mechanisms of action and target 


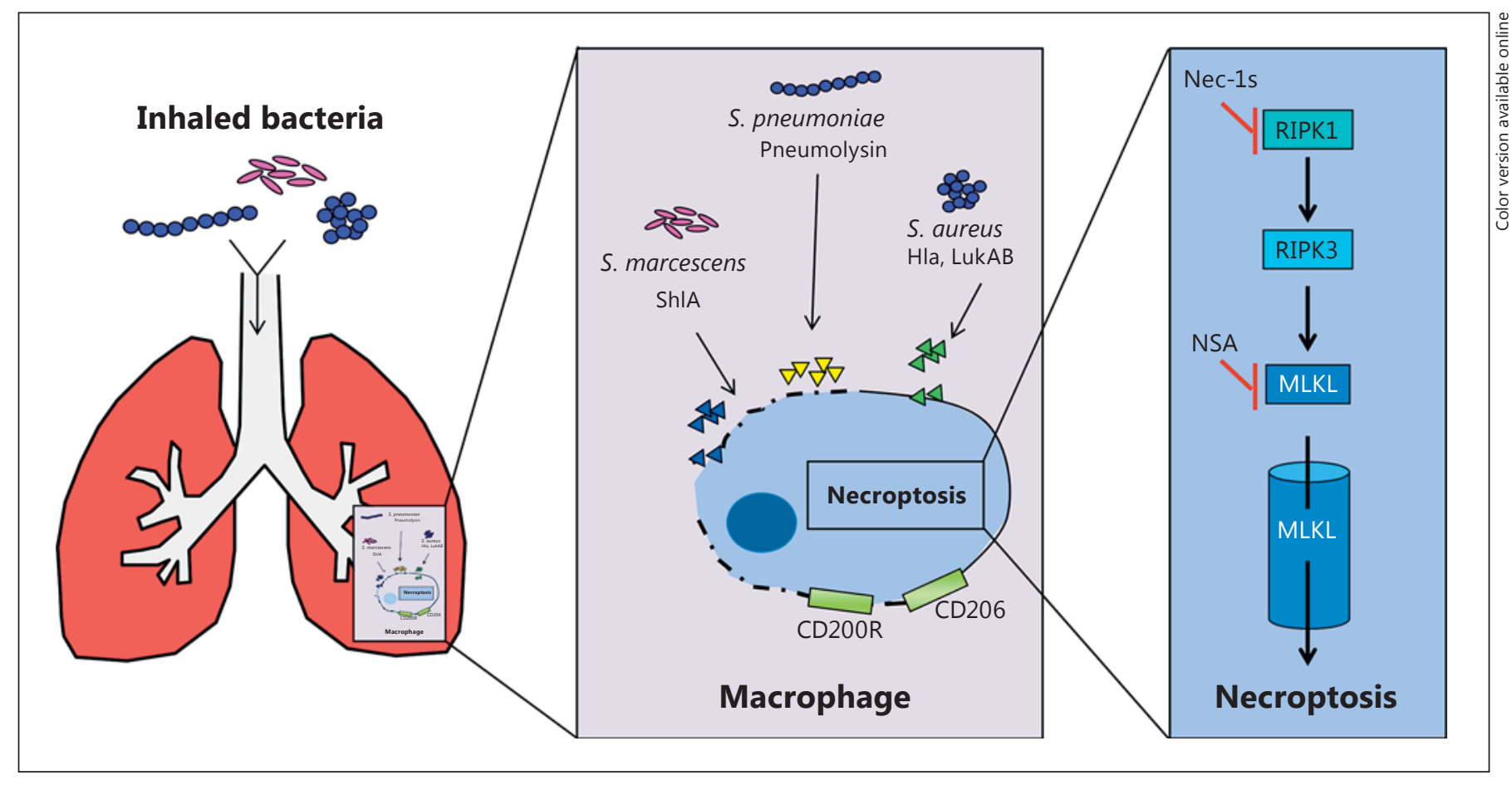

Fig. 3. Mechanisms of necroptosis. Inhaled bacteria that secrete toxins initiate necroptotic pathways in the macrophages of the lung. This can lead to the death of the immune cell population, with or without the bacteria, and also the loss of anti-inflammatory regulation if immunosuppressive cells are lost (in the case of resident alveolar macrophages that express CD200R and CD206). Necroptosis is induced in macrophages, with the final effector being the formation of the MLKL multimer pore. Nec-1s, necrostatin-1. macrophages. However, for S. aureus, macrophage loss through necroptosis is highly deleterious as it eliminates an immunomodulatory effector; the macrophages activated by $S$. marcescens necroptosis contribute to excessive inflammation, therefore blocking necroptosis and significantly improving the outcome. The loss of resident alveolar macrophages that have a predominantly anti-inflammatory function contributes to dysregulated inflammation and impairs bacterial clearance. Mechanisms of necroptotic signaling may be involved, even for organisms that do not activate a brisk inflammatory response such as CRKP. Our findings show that, in the absence of pore-forming toxin production, attenuating the necroptosis (at least in a ipk $^{-/-}$mouse) promotes bacterial clearance and improves host outcome.

There is much more to learn about necroptosis and its pro- versus anti-inflammatory effects in the lung. What we know now is that the host response is highly dependent upon the etiology of the infection. For most airway pathogens, there is a delicate balance of the induction of inflammatory signaling sufficient for bacterial clearance and that associated with lung damage that will results in impaired oxygen exchange and, ultimately, death. Although we have focused upon the role of necroptosis in bacterial infection, there is substantially greater interest in its immunoregulatory role in autoimmune diseases and inflammatory bowel disease. While effective inhibitors of the components of the necroptosis pathway are being developed for several clinical indications, it is important to recognize that inhibiting necroptosis is very likely to have effects on the pathogenesis of common infections, particularly pneumonia.

\section{Acknowledgements}

This work was supported by NIH RO1 HL073989 to A.P. and NIH K12HD047349-08 to D.A.

\section{Disclosure Statement}

The authors declare no competing financial interests. 


\section{References}

1 Galluzzi L, Buque A, Kepp O, Zitvogel L, Kroemer G: Immunogenic cell death in cancer and infectious disease. Nat Rev Immunol 2016, E-pub ahead of print.

2 Kerr JF, Wyllie AH, Currie AR: Apoptosis: a basic biological phenomenon with wideranging implications in tissue kinetics. $\mathrm{Br} \mathrm{J}$ Cancer 1972;26:239-257.

3 Thompson CB: Apoptosis in the pathogenesis and treatment of disease. Science 1995;267: 1456-1462.

4 Koopman G, Reutelingsperger CP, Kuijten GA, Keehnen RM, Pals ST, van Oers MH: Annexin $\mathrm{V}$ for flow cytometric detection of phosphatidylserine expression on B cells undergoing apoptosis. Blood 1994;84:1415-1420.

5 Clouston WM, Kerr JF: Apoptosis, lymphocytotoxicity and the containment of viral infections. Med Hypotheses 1985;18:399-404.

6 Robaye B, Mosselmans R, Fiers W, Dumont JE, Galand P: Tumor necrosis factor induces apoptosis (programmed cell death) in normal endothelial cells in vitro. Am J Pathol 1991; 138:447-453.

7 Linkermann A, Green DR: Necroptosis. N Engl J Med 2014;370:455-465.

8 Newton K: RIPK1 and RIPK3: critical regulators of inflammation and cell death. Trends Cell Biol 2015;25:347-353.

9 Degterev A, Hitomi J, Germscheid M, Ch'en IL, Korkina O, Teng X, Abbott D, Cuny GD, Yuan C, Wagner G, Hedrick SM, Gerber SA, Lugovskoy A, Yuan J: Identification of RIP1 kinase as a specific cellular target of necrostatins. Nat Chem Biol 2008;4:313-321.

10 Sridharan H, Upton JW: Programmed necrosis in microbial pathogenesis. Trends Microbiol 2014;22:199-207.

11 Kim J, Kwak HJ, Cha JY, Jeong YS, Rhee SD, Kim KR, Cheon HG: Metformin suppresses lipopolysaccharide (LPS)-induced inflammatory response in murine macrophages via activating transcription factor-3 (ATF-3) induction. J Biol Chem 2014;289:23246-23255.

12 Mizumura K, Maruoka S, Gon Y, Choi AM, Hashimoto S: The role of necroptosis in pulmonary diseases. Respir Invest 2016;54:407412.

13 Dondelinger Y, Declercq W, Montessuit S, Roelandt R, Goncalves A, Bruggeman I, Hulpiau P, Weber K, Sehon CA, Marquis RW, Bertin J, Gough PJ, Savvides S, Martinou J-C, Bertrand M, Vandenabeele P: MLKL compromises plasma membrane integrity by binding to phosphatidylinositol phosphates. Cell Rep 2014;7:971-981.

14 Murphy JM, Czabotar PE, Hildebrand JM, Lucet IS, Zhang JG, Alvarez-Diaz S, Lewis R, Lalaoui N, Metcalf D, Webb AI, Young SN, Varghese LN, Tannahill GM, Hatchell EC, Majewski IJ, Okamoto T, Dobson RC, Hilton DJ, Babon JJ, Nicola NA, Strasser A, Silke J, Alexander WS: The pseudokinase MLKL mediates necroptosis via a molecular switch mechanism. Immunity 2013;39:443-453.
15 Monie TP, Bryant CE: Caspase- 8 functions as a key mediator of inflammation and pro-IL$1 \beta$ processing via both canonical and noncanonical pathways. Immunol Rev 2015;265: $181-193$.

16 Kang TB, Yang SH, Toth B, Kovalenko A, Wallach D: Caspase- 8 blocks kinase RIPK3mediated activation of the NLRP3 inflammasome. Immunity 2013;38:27-40.

17 Najjar M, Saleh D, Zelic M, Nogusa S, Shah S, Tai A, Finger JN, Polykratis A, Gough PJ, Bertin J, Whalen MJ, Pasparakis M, Balachandran S, Kelliher M, Poltorak A, Degterev A: RIPK1 and RIPK3 kinases promote celldeath-independent inflammation by Toll-like receptor 4 . Immunity 2016;45:46-59.

18 Chan FK, Luz NF, Moriwaki K: Programmed necrosis in the cross talk of cell death and inflammation. Annu Rev Immunol 2015;33:79106.

19 Dannappel M, Vlantis K, Kumari S, Polykratis A, Kim C, Wachsmuth L, Eftychi C, Lin J, Corona T, Hermance N, Zelic M, Kirsch P, Basic M, Bleich A, Kelliher M, Pasparakis M: RIPK1 maintains epithelial homeostasis by inhibiting apoptosis and necroptosis. Nature 2014;513:90-94.

20 Liu X, Shi F, Li Y, Yu X, Peng S, Li W, Luo X, Cao Y: Post-translational modifications as key regulators of TNF-induced necroptosis. Cell Death Dis 2016;7.

21 Green DR, Oberst A, Dillon CP, Weinlich R, Salvesen GS: RIPK-dependent necrosis and its regulation by caspases: a mystery in five acts. Mol Cell 2011;44:9-16.

22 Moquin DM, McQuade T, Chan FK: CYLD deubiquitinates RIP1 in the TNFa-induced necrosome to facilitate kinase activation and programmed necrosis. PLoS One 2013;8: e76841.

23 Moriwaki K, Balaji S, McQuade T, Malhotra N, Kang J, Chan FK: The necroptosis adaptor RIPK3 promotes injury-induced cytokine expression and tissue repair. Immunity $2014 ; 41$ : 567-578.

24 Bleriot C, Lecuit M: The interplay between regulated necrosis and bacterial infection. Cell Mol Life Sci 2016;73:2369-2378.

25 Kitur K, Parker D, Nieto P, Ahn DS, Cohen TS, Chung S, Wachtel S, Bueno S, Prince A: Toxin-induced necroptosis is a major mechanism of Staphylococcus aureus lung damage. PLoS Pathog 2015;11:e1004820.

26 Lawlor KE, Khan N, Mildenhall A, Gerlic M, Croker BA, D’Cruz AA, Hall C, Kaur Spall S, Anderton H, Masters SL, Rashidi M, Wicks IP, Alexander WS, Mitsuuchi Y, Benetatos CA, Condon SM, Wong WW, Silke J, Vaux DL, Vince JE: RIPK3 promotes cell death and NLRP3 inflammasome activation in the absence of MLKL. Nat Commun 2015;6:6282.
27 Kayagaki N, Stowe IB, Lee BL, O’Rourke K, Anderson K, Warming S, Cuellar T, Haley B, Roose-Girma M, Phung QT, Liu PS, Lill JR, Li $\mathrm{H}, \mathrm{Wu}$ J, Kummerfeld S, Zhang J, Lee WP, Snipas SJ, Salvesen GS, Morris LX, Fitzgerald L, Zhang Y, Bertram EM, Goodnow CC, Dixit VM: Caspase-11 cleaves gasdermin D for non-canonical inflammasome signalling. Nature 2015;526:666-671.

28 Greenlee-Wacker MC, Rigby KM, Kobayashi SD, Porter AR, DeLeo FR, Nauseef WM: Phagocytosis of Staphylococcus aureus by human neutrophils prevents macrophage efferocytosis and induces programmed necrosis. J Immunol 2014;192:4709-4717.

29 Robinson N, McComb S, Mulligan R, Dudani R, Krishnan L, Sad S: Type I interferon induces necroptosis in macrophages during infection with Salmonella enterica serovar $\mathrm{Ty}$ phimurium. Nat Immunol 2012;13:954-962.

30 Chow SH, Deo P, Naderer T: Macrophage cell death in microbial infections. Cell Microbiol 2016;18:466-474.

31 Ch'en IL, Tsau JS, Molkentin JD, Komatsu M, Hedrick SM: Mechanisms of necroptosis in T cells. J Exp Med 2011;208:633-641.

32 Li S, Zhang L, Yao Q, Li L, Dong N, Rong J, Gao W, Ding X, Sun L, Chen X, Chen S, Shao F: Pathogen blocks host death receptor signalling by arginine GlcNAcylation of death domains. Nature 2013;501:242-246.

33 Roca FJ, Ramakrishnan L: TNF dually mediates resistance and susceptibility to mycobacteria via mitochondrial reactive oxygen species. Cell 2013;153:521-534.

34 Gonzalez-Juarbe N, Gilley RP, Hinojosa CA, Bradley KM, Kamei A, Gao G, Dube PH, Bergman MA, Orihuela CJ: Pore-forming toxins induce macrophage necroptosis during acute bacterial pneumonia. PLoS Pathog 2015;11:e1005337.

35 Parker D, Prince A: Staphylococcus aureus induces type I IFN signaling in dendritic cells via TLR9. J Immunol 2012;189:4040-4046.

36 Parker D, Planet PJ, Soong G, Narechania A, Prince A: Induction of type I interferon signaling determines the relative pathogenicity of Staphylococcus aureus strains. PLoS Pathog 2014; 10:e1003951.

37 Duprez L, Takahashi N, Van Hauwermeiren F, Vandendriessche B, Goossens V, Vanden Berghe T, Declercq W, Libert C, Cauwels A, Vandenabeele P: RIP kinase-dependent necrosis drives lethal systemic inflammatory response syndrome. Immunity 2011;35:908-918.

38 Rickard JA, O’Donnell JA, Evans JM, Lalaoui N, Poh AR, Rogers T, Vince JE, Lawlor KE, Ninnis RL, Anderton H, Hall C, Spall SK, Phesse TJ, Abud HE, Cengia LH, Corbin J, Mifsud S, Di Rago L, Metcalf D, Ernst M, Dewson G, Roberts AW, Alexander WS, Murphy JM, Ekert PG, Masters SL, Vaux DL, Croker BA, Gerlic M, Silke J: RIPK1 regulates RIPK3-MLKL-driven systemic inflammation and emergency hematopoiesis. Cell 2014;157:1175-1188. 
39 Kitur K, Wachtel S, Brown A, Wickersham M, Paulino F, Penaloza HF, Soong G, Bueno S, Parker D, Prince A: Necroptosis promotes Staphylococcus aureus clearance by inhibiting excessive inflammatory signaling. Cell Rep 2016;16:2219-2230.

40 Regueiro V, Moranta D, Frank CG, Larrarte E, Margareto J, March C, Garmendia J, Bengoechea JA: Klebsiella pneumoniae subverts the activation of inflammatory responses in a NOD1-dependent manner. Cell Microbiol 2011;13:135-153.
41 Xiong H, Carter RA, Leiner IM, Tang YW, Chen L, Kreiswirth BN, Pamer EG: Distinct contributions of neutrophils and CCR2+ monocytes to pulmonary clearance of different Klebsiella pneumoniae strains. Infect Immun 2015;83:3418-3427.

42 Ahn D, Peñaloza H, Wang Z, Wickersham M, Parker D, Patel P, Koller A, Chen E, Bueno S, Uhlemann A, Prince A: Acquired resistance to innate immune clearance promotes Klebsiella pneumoniae ST258 pulmonary infection. JCI Insight 2016;1:e89704.
43 Gomez-Simmonds A, Greenman M, Sullivan SB, Tanner JP, Sowash MG, Whittier S, Uhlemann AC: Population structure of Klebsiella pneumoniae causing bloodstream infections at a New York City tertiary care hospital: diversification of multidrug-resistant isolates. J Clin Microbiol 2015;53:2060-2067.

44 Paczosa MK, Mecsas J: Klebsiella pneumoni$a e$ : going on the offense with a strong defense. Microbiol Mol Biol Rev 2016;80:629-661.

45 Parker D, Prince A: Type I interferon response to extracellular bacteria in the airway epithelium. Trends Immunol 2011;32:582588 